\title{
Editorial
}

\section{Barrow Ruptured Aneurysm Trial}

\author{
Andrew Molyneux, F.R.C.R., ${ }^{1}$ Richard KerR, F.R.C.S., ${ }^{1}$ \\ AND JACQUELINE BIRKS, M.SC. ${ }^{2}$
}

${ }^{1}$ Oxford Neurovascular and Neuroradiology Research Unit, Nuffield Department of Surgical Sciences, University of Oxford, and ${ }^{2}$ Centre for Statistics in Medicine, University of Oxford, Wolfson College, Oxford, United Kingdom

We are grateful to the editor for this opportunity to comment on the report of the 3-year outcomes of the Barrow Ruptured Aneurysm Trial (BRAT). ${ }^{15}$

Before making specific comments on the trial it may be helpful to readers to set out the widely accepted standards and procedures for the conduct design and reporting of randomized clinical trials. These are set down in the Consolidated Standards of Reporting Trials (CONSORT) ${ }^{1,14}$ These criteria have been adopted by more than 300 international medical journal editors including all the major medical journals.

The purpose of the CONSORT criteria is to ensure that studies published in these journals fulfill methodological rigor to ensure the quality and scientific reliability of the published data.

Three key requirements of CONSORT are:

1) Publication of the full protocol, and any later modifications, before and during the trial. Most importantly this must include any proposed subgroups (preplanned subgroup analysis).

2) Registration and protocol submission to a central database of randomized clinical trials such as Clinical Trials.gov.

3) Full accounting for all enrolled patients including those lost to follow-up and reporting of all deaths. Reporting the extent of missing patient data is essential.

\section{Methodological Issues}

There are a number of design and reporting features of BRAT that do not fulfill CONSORT criteria, which have exposed the design and methodological weaknesses of the trial.

The authors of the current paper state that the protocol was described in their original report. ${ }^{5}$ This is not correct, as the full BRAT protocol to our knowledge has not been published, and it would be very helpful if the protocol was in the public domain and accessible. BRAT was not registered with any of the clinical trial databases until April 2012, sometime after its completion of recruitment and the first publication (Clincaltrials.gov 30 April 2012 NCT01593267). The importance of both of these, as CONSORT requirements, is to specify any planned sub- group or additional analyses. Prespecifying subgroups is essential because, as many readers will know, post hoc subgroup analysis is not a valid scientific technique, and the reliability of the results of such analyses must be treated with considerable caution. The current paper not only appears to use subgroups that have not been prespecified but also has a substantial number of missing outcomes. Additional analyses excluding patients who received no treatment and analyses based on treatment actually received are reported but do not appear to have been prespecified. This makes any conclusions drawn from this report scientifically unreliable.

One significant problem of this trial was that $30 \%$ of randomized patients did not receive the allocated treatment, and this has resulted in a serious imbalance between treatment groups.

From a clinical trial methodological point of view this indicates that the original trial inclusion criteria were inappropriate for a randomized trial. In International Subarachnoid Aneurysm Trial (ISAT), patients needed to have an aneurysm and it had to be judged suitable for both treatments. In BRAT, patients unsuitable for either treatment (no aneurysm present) and patients unsuitable for one of the allocations (unsuitable aneurysm anatomy for coil embolization) were enrolled. A primary principle of randomized trials is that patients should be potentially eligible for either treatment. This represents a significant deficiency in the design of the trial and gives a false impression that this trial, as reported, was able to properly enroll a large proportion of the subarachnoid hemorrhage (SAH) patient population.

\section{The Present Analysis of the Existing Data}

To be valid, a randomized trial must be analyzed by intention to treat. This means including all the original patients in the analysis according to randomization allocation, whether they crossed over or received no treatment. The results of the trial then apply to the population randomized. Although the authors analyze by intention to treat for the 1-year data, unfortunately, in the case of the 3 -year data, there is no methodological basis for the analysis; it is an ad hoc analysis. The analysis as reported, by treatment actually received, indicates that the investigators are acknowledging problems of the trial design, although this is not the solution. The results, after analyzing according to treatment received, are biased, because the groups are no longer balanced by randomization, and meaningful interpretation is not possible.

In the reporting of trials it is very important that missing data are clearly recorded and accounted for and that it is made explicit how the missing outcomes are distributed between the allocations. 
In this report and in the first BRAT paper, the extent of the missing outcomes is not explicitly stated. Of the total of 471 patients enrolled, 403 had outcome data reported (Table 3$)^{5}$-about $13 \%$ missing. In the present paper the authors are able to report 349 of 408 outcomes for treated patients at 3 years (Table 1) -about 15\% missing.

It would be usual when reporting a clinical trial to state the distribution of these missing outcomes between the groups and to know how many deaths there are in each allocation. There may be significant imbalances between the groups in the extent of the missing data that could seriously affect the results.

Without these data, any conclusions drawn may be unreliable. It would be very helpful to have tables of the individual outcomes and deaths listed and the exact distribution of this missing data, and whether this included all enrolled patients or just treated patients.

\section{Misleading Statements Regarding ISAT}

The authors make a number of statements in the current paper that are misleading and factually inaccurate regarding ISAT, ${ }^{9,10}$

Throughout the paper, the authors cite as fact that $80 \%$ of eligible patients were not enrolled in ISAT. Whilst it is correct that about $80 \%$ of all patients who were admitted to ISAT centers during the recruitment period of the trial with a confirmed aneurysmal SAH (aSAH) were not enrolled, many potentially eligible patients were not enrolled for operational reasons, such as no interventionist available to treat the patient, no angiogram room available, unsuitable aneurysm anatomy, or lack of consent. The trial protocol, published by The Lancet in 1997, stated clearly that the patient had to have an aneurysm suitable for both treatments and clinical equipoise existed for these patients. The proportion of aSAH patients enrolled in individual centers varied widely between $1 \%$ and $44 \%$, reflecting individual clinical views and belief at the time that a particular treatment was better (that is, lack of clinical equipoise). We have addressed this widespread fallacy in a recent letter published in the Journal of Neurosurgery. ${ }^{6}$

The statement suggesting that there was selection bias in ISAT. We have always stated in all the ISAT-related publications that it is a study of a selected population of aSAH patients. It is not correct to call this bias. The selected population is fully described; within that population the allocation was concealed, and reporting was by intention to treat. Once patients were enrolled there was no bias in the trial. The population of patients in ISAT was balanced for known prognostic factors by the minimization process, with the allocation group revealed after the patient's baseline data had been collected. In practice, $97 \%$ of the patients had anterior circulation aneurysms, 88\% were in good grade (World Federation of Neurosurgical Societies [WFNS] Grade I or II), and $90 \%$ had aneurysms less than $10 \mathrm{~mm}$ in diameter. In the United Kingdom (UK) national audit data this represented about $80 \%$ of more than 2000 patients treated between 2001 and 2002 in the UK. ${ }^{4,13}$ The ISAT patient population inevitably shows some differences from BRAT.
They state that the outcomes in ISAT are similar in the 2 groups at 5 years. In ISAT this was the case if the patients were alive. It ignores the fact that there was a significant excess mortality in the clip-treated group at 5 years after treatment. The similarity of outcome with respect to dependence in the report was conditional on patient survival. This was clearly stated in the 2009 Lancet Neurology paper. ${ }^{8}$ Therefore, the bald statement they make in this respect that the late outcomes are similar is not valid as stated.

In the Discussion, the authors reference the paper of Gnanalingham and colleagues ${ }^{3}$ as being from a major ISAT participating institution. This is incorrect, the data used for this paper were not from an ISAT recruiting center.

The authors stated in their response to the letter by Drs. Darsaut and Raymond ${ }^{2}$ that although the ISAT's ratio of screened to enrolled patients was a common criticism, they "did not suggest that it invalidated the findings with respect to the population studied or even that it was a valid criticism." This current paper appears to contradict that statement.

\section{Comments Regarding the Discussion}

The Discussion cites O'Kelly and colleagues' ${ }^{11}$ study of cases in the Ontario Stroke database involving patients treated between 1995 and 2004 as evidence that there were worse outcomes in the coil-treated patients. This presupposes that the characteristics of the population in the cliptreated and coil-treated groups have similar baseline clinical characteristics, which of course they could not have because they were not randomized. Whilst the authors attempted to stratify for initial condition in that paper and adjust statistically for the inevitable differences between the groups, there were insufficient clinical data available from an administrative database such as this to stratify for major prognostic features-notably, even clinical grade on admission. For example, a significantly higher proportion of patients treated with coil embolization were ventilated on admission, and O'Kelly et al. recognized these shortcomings. Thus, attempting to draw scientifically valid conclusions from such data may be misleading.

A larger administrative database study was published by Qureshi and colleagues, ${ }^{12}$ who used the US National Inpatient Sample database. Their paper compared the inhospital mortality in the years 2000-2002 and 2004-2006 on the basis of a total sample size of 147,000 patients. It showed a $3 \%$ fall in in-hospital mortality and an increase in the rate of treatment with coil embolization from $3 \%$ to $17 \%$ of patients with SAH.

We appreciate that the BRAT investigators have made a significant effort to address what was widely perceived to be a weakness of ISAT. BRAT was originally designed as a pilot study to test the feasibility of a larger trial, and in the report of the 1-year results, the authors stated that the study was not expected to be powered sufficiently to demonstrate differences in outcome between the groups. ${ }^{5}$ However in that report, despite the extent of the missing outcomes, the primary outcome, based on intention to treat, showed a larger absolute benefit of coiling $-10 \%$ 
compared with the $7.4 \%$ that was shown in ISAT, and this did reach statistical significance at a $5 \%$ level $(p=0.02)$.

The current paper attempts to contradict their original findings, using subgroup analyses that have not been prespecified in a published protocol, whilst having a substantial number of missing outcomes, and attempts to draw conclusions on an inadequate sample size. It does not conform to the standard reporting criteria, set out in the CONSORT criteria, whilst containing a number of factually inaccurate statements concerning ISAT. The present paper is scientifically flawed.

(http://thejns.org/doi/abs/10.3171/2012.11.JNS121406)

\section{Disclosure}

The authors report no conflict of interest.

\section{References}

1. Begg C, Cho M, Eastwood S, Horton R, Moher D, Olkin I, et al: Improving the quality of reporting of randomized controlled trials. The CONSORT statement. JAMA 276:637-639, 1996

2. Darsaut TE, Raymond J: Barrow Ruptured Aneurysm Trial. J Neurosurg 117:378-379, 2012 (Letter)

3. Gnanalingham KK, Apostolopoulos V, Barazi S, O’Neill K: The impact of the International Subarachnoid Aneurysm Trial (ISAT) on the management of aneurysmal subarachnoid haemorrhage in a neurosurgical unit in the UK. Clin Neurol Neurosurg 108:117-123, 2006

4. Langham J, Reeves BC, Lindsay KW, van der Meulen JH, Kirkpatrick PJ, Gholkar AR, et al: Variation in outcome after subarachnoid hemorrhage: a study of neurosurgical units in the UK and Ireland. Stroke 40:111-118, 2009

5. McDougall CG, Spetzler RF, Zabramski JM, Partovi S, Hills NM, Nakaji P: The Barrow Ruptured Aneurysm Trial. Clinical article. J Neurosurg 116:135-144, 2012

6. Molyneux AJ, Kerr RSC: The Barrow Ruptured Aneurysm Trial and International Subarachnoid Aneurysm Trial. J Neurosurg 118:478-480, 2013 (Letter)

7. Molyneux AJ, Kerr RSC: Protocol 99PRT18 International Subarachnoid Aneurysm Trial (ISAT). (http://www. thelancet.com/protocol-reviews/99PRT-18) [Accessed March 21, 2013]

8. Molyneux AJ, Kerr RSC, Birks J, Ramzi N, Sneade M, Rischmiller J, et al: Risk of recurrent subarachnoid haemorrhage, death and the standardised mortality ratios after clipping or coiling of an intracranial aneurysm the International Subarachnoid Aneurysm Trial (ISAT): long-term follow-up. Lancet Neurol 8:427-433, 2009

9. Molyneux AJ, Kerr RSC, Stratton P, Clarke M, Shrimpton J, Holmar R, et al: International Subarachnoid Aneurysm Trial (ISAT) of neurosurgical clipping versus endovascular coiling in 2143 patients with ruptured intracranial aneurysms: a randomized trial. Lancet 360:1267-1274, 2002

10. Molyneux AJ, Kerr RSC, Yu LM, Clarke M, Sneade M, Yarnold JA, et al: International Subarachnoid Aneurysm Trial (ISAT) of neurosurgical clipping versus endovascular coiling in 2143 patients with ruptured intracranial aneurysms: a randomised comparison of effects on survival, dependency, seizures, rebleeding, subgroups, and aneurysm occlusion. Lancet 366:809-817, 2005

11. O'Kelly CJ, Kulkarni AV, Austin PC, Wallace MC, Urbach D: The impact of therapeutic modality on outcomes following repair of ruptured intracranial aneurysms: an administrative data analysis. Clinical article. J Neurosurg 113:795-801, 2010
12. Qureshi AI, Vazquez G, Tariq N, Suri MF, Lakshminarayan K, Lanzino G: Impact of International Subarachnoid Aneurysm Trial results on treatment of ruptured intracranial aneurysms in the United States. Clinical article. J Neurosurg 114: 834-834, 2011

13. Royal College of Surgeons of England: National Study of Subarachnoid Haemorrhage Final Report 2006. London: The Royal College of Surgeons of England (http://www.rcseng.ac. uk/surgeons/research/surgical-research/docs/National\%20 Study $\% 20$ of $\% 20$ Subarahnoid $\% 20$ Haemorrhage $\% 20$ Final $\% 20$ Report\%202006.pdf) [Accessed March 21, 2013]

14. Shultz KF, Altman DG, Moher D: CONSORT 2010 statement: updated guidelines for reporting parallel group randomised trials. Int J Surg 9:672-677, 2011

15. Spetzler RF, McDougall CG, Albuquerque FC, Zabramski JM, Hills NK, Partovi S, et al: The Barrow Ruptured Aneurysm Trial: 3-year results. Clinical article. J Neurosurg [epub ahead of print April 26, 2013. DOI: 10.3171/2013.3.JNS12683]

\section{Response}

Robert F. Spetzler, M.D., ${ }^{1}$ Cameron G. McDougall, M.D., Felipe C. Albuquerque, M.D., ${ }^{1}$ Joseph M. Zabramski, M.D., ${ }^{1}$ Nancy K. Hills, Ph.D., ${ }^{2,3}$ Shahram Partovi, M.D., ${ }^{4}$ Peter NaKaji, M.D., ${ }^{1}$ And Robert C. Wallace, M.D. ${ }^{4}$

Divisions of ${ }^{1}$ Neurological Surgery, and ${ }^{4}$ Neuroradiology, Barrow Neurological Institute, St. Joseph's Hospital and Medical Center, Phoenix, Arizona; and Departments of ${ }^{2}$ Neurology and ${ }^{3}$ Epidemiology and Biostatistics, University of California, San Francisco, California

Note: Italicized comments are direct quotations from Dr. Molyneux and colleagues' editorial; the reference cited in those quotations can be found at the end of his editorial.

We appreciate and thank the authors for the obvious amount of time and thought that has gone into this editorial and will attempt to address the major concerns they have raised. Given the lack of consensus that still exists, a fair and robust dialogue regarding the management of patients with aSAH is appropriate and necessary. As strong advocates for randomized trials to answer difficult management questions, we concur that it is equally important to scrutinize data for consistency and applicability.

Additional analyses excluding patients who received no treatment and analyses based on treatment actually received are reported but do not appear to have been prespecified. This makes any conclusions drawn from this report scientifically unreliable.

We agree that analyses based on subgroups that were not prespecified must be viewed as secondary analyses and interpreted with caution as a result. However, we felt that the exclusion of patients who had not received treatment was justified for several reasons. As we stated in our paper, "Although our prior analysis of outcomes at Year 1 included these untreated patients, they have been excluded from the analysis of outcomes at Year 3, given that these outcomes cannot be associated with a treatment that did not occur." In order to address concerns that this might raise, we performed sensitivity analyses to determine whether exclusion of these nontreated patients 
would affect our results. As stated, nontreated patients who were randomized to clipping and coil embolization were equally matched: there were 3 deaths in each group, and each group had the same clinical outcomes $(p=1.0)$. Our rationale was presented in the paper as follows: "Although our analysis of outcomes at 1 year included patients who were randomized but not treated (due to nonaneurysmal SAH or death before treatment), these patients were excluded from the current analysis for 2 reasons. First, there was no difference in the outcomes between them at 3 years. Second, they were not treated and therefore were not subject to the decision to treat as assigned or cross over to the alternate treatment."

Finally, as demonstrated in Table 1, after exclusion of the nontreated cohort, the 1-year results remain statistically significant in favor of coiling at a $p$ value $(0.03)$ similar to that obtained when they were included $(p=0.02)$.

We perceive the crossover decision-making process as being very important, and examining this issue was one of the primary goals of BRAT; excluding those patients for no treatment decision was possible because they were dead or had no aneurysm was therefore deemed appropriate.

One significant problem of this trial was that $30 \%$ of randomized patients did not receive the allocated treatment, and this has resulted in a serious imbalance between treatment groups.

In fact, nearly all patients received their allocated treatment, given a policy of clipping versus coiling as the first choice of therapy.

A primary principle of randomized trials is that patients should be potentially eligible for either treatment. This represents a significant deficiency in the design of the trial and gives a false impression that this trial, as reported, was able to properly enroll a large proportion of the SAH patient population.

What is characterized as a deficiency served a very legitimate purpose, one complementary to ISAT. It remained unclear from ISAT what proportion of ruptured aneurysms might legitimately be considered for coil embolization. While only approximately $20 \%$ of potentially eligible patients in ISAT centers were actually enrolled, the reasons that nearly $80 \%$ of patients were not enrolled have not been specified. Therefore, it remains uncertain whether the patients actually enrolled in ISAT are representative of the general population of patients with ruptured aneurysms. The ISAT results apply to patients with similar aneurysms in similar centers but otherwise are less amenable to generalization. Nonetheless, that has not kept its results from being improperly generalized. By contrast, BRAT was designed as a pilot trial where a policy of prioritizing one treatment over the other allowed more comprehensive inclusion of patients with ruptured aneurysms. In this setting it became apparent that at least $62 \%$ of aneurysms could be treated by coil embolization, with clinical outcomes as good as, if not better than, surgical clipping (depending on the time frame of the analysis). It remains unanswered whether the results would differ if a higher proportion of aneurysms had actually been treated with coils, or whether similar results would be seen in other centers. This was clearly described in the original publication, ${ }^{5}$ and reiterated in the response to a subsequent letter to the editor. ${ }^{2}$

The claim that virtually all aneurysms can be treated endovascularly is frequently stated at international conferences (something Molyneux and colleagues suggest as well in their editorial, based on their comments that $80 \%$ of more than 2000 patients in the UK database have baseline characteristics similar to those in ISAT), with the assumption being that equipoise is clearly present. Therefore, it is unclear how including all SAH patients can lead to the "false impression that this trial, as reported, was able to properly enroll a large proportion of the SAH patient population." It is precisely BRAT's all-inclusiveness that gives it the ability to address the majority of the SAH patient population as opposed to a subgroup of that population. Part of the aim of the BRAT trial was to determine the percentage of patients with a SAH that experienced physicians either felt would be appropriate for the assigned treatment or should be crossed over. Clearly that number cannot be available before the trial is undertaken and provides an important contribution in the management of SAH. Even when randomization occurs just prior to initiating treatment, as in ISAT, crossovers still occur. Therefore, even in ISAT, there is a difference that is crossover dependent: "There was a non-significant increased risk of rebleeding from the treated aneurysm in the endovascular cohort $(\log$ rank $\mathrm{p}=0.06)$ by intention-to-treat analysis and a significant difference when analysis was by

TABLE 1: Patients with mRS scores $>2$ across BRAT follow-up by assigned treatment*

\begin{tabular}{|c|c|c|c|c|c|c|}
\hline Time Point & $\begin{array}{c}\text { Available for } \\
\text { Analysis }\end{array}$ & $\begin{array}{c}\text { Coil-Assigned } \\
(\mathrm{mRS}>2)\end{array}$ & Clip-Assigned (mRS >2) & OR & $95 \% \mathrm{Cl}$ & $p$ Value \\
\hline randomization & 408 & 199 & 209 & & & \\
\hline discharge & 406 & 127/198 (64.1) & 147/208 (70.7) & 1.35 & $0.89-2.05$ & 0.16 \\
\hline 6 mos & 341 & 40/171 (23.4) & $62 / 170(36.5)$ & 1.88 & $1.18-3.03$ & 0.009 \\
\hline $1 \mathrm{yr}$ & 358 & 42/174 (24.1) & 64/184 (34.8) & 1.68 & $1.06-2.67$ & 0.03 \\
\hline 3 yrs & 349 & $51 / 170(30.0)$ & $64 / 179(35.8)$ & 1.30 & $0.83-2.04$ & 0.25 \\
\hline $3 \mathrm{yr}-\mathrm{CF} \dagger$ & 366 & 51/178 (28.7) & 64/188 (34.0) & 1.29 & $0.83-2.00$ & 0.27 \\
\hline
\end{tabular}

\footnotetext{
* Values represent numbers of patients (\%) unless otherwise indicated. Odds ratios, confidence intervals, and $p$ values were obtained from unadjusted logistic regression. $\mathrm{mRS}=$ modified Rankin Scale.

$\dagger \mathrm{CF}=$ carry forward. Includes patients seen at 1 year but not at 3 years.
} 
actual treatment received $(\log$ rank $\mathrm{p}=0 \cdot 02) .{ }^{\prime \prime}{ }^{\circ}$ Despite the concerns expressed in the ISAT-accompanying editorial ${ }^{7}$ about restricting the ISAT results to the limited cohort studied, this has not happened in the real world, as shown in the change of practice patterns. ${ }^{3}$ This is precisely why a trial that is as inclusive as possible has significant value.

The results, after analyzing according to treatment received, are biased, because the groups are no longer balanced by randomization, and meaningful interpretation is not possible.

Although this has been addressed above, Table 1 in our publication shows the results excluding the pretreatment cohort, while Table 2 is a new table that includes all randomized patients.

No significant difference was observed between analyses conducted with or without the pretreatment cohort.

In this report and in the first BRAT paper the extent of the missing outcomes is not explicitly stated. Of the total of 471 patients enrolled, 403 had outcome data reported (Table 3$)^{5}-$ about $13 \%$ missing. In the present paper the authors are able to report 349 of 408 outcomes for treated patients at 3 years (Table 1)-about $15 \%$ missing.

More precisely, the percentage of patients missing data is $14 \%$ in each of the 2 periods $(100-[403 / 471 \times$ $100]$ and $100-[349 / 408 \times 100])$. In order to minimize possible bias due to data missing from patients at 3 years who were included in the 1-year analysis (when coiling had a better outcome), we conducted a sensitivity analysis in which the last modified Rankin Scale (mRS) score of this group of patients was carried forward. In this way, we examined the possibility that the missing data influenced the results. When the carried-forward data are included, only $10 \%$ of treated patients are not accounted for, and there is no appreciable difference in the results. While every effort was made to get as complete a follow-up as possible, inevitably some patients remain lost to follow-up.

It would be usual when reporting a clinical trial to state the distribution of these missing outcomes between the groups and to know how many deaths there are in each allocation. There may be significant imbalances between the groups in the extent of the missing data that could seriously affect the results.

Deaths are listed in Table 3, which shows additional information on deaths in the 2 groups (which we had included in our initial drafts, but which were shortened to be more concise).

\section{Regarding "Misleading Statements"}

Throughout the paper the authors cite as fact that $80 \%$ of eligible patients were not enrolled in ISAT. Whilst it is correct that about $80 \%$ of all patients that were admitted to ISAT centers during the recruitment period of the trial with a confirmed aneurysmal SAH (aSAH) were not enrolled, many potentially eligible patients were not enrolled for operational reasons, such as no interventionist available to treat the patient, no angiogram room available, unsuitable aneurysm anatomy, or lack of consent. The trial protocol, published by The Lancet
TABLE 2: Patients with $m R S$ scores $>2$ for 3 -year follow-up including all randomized patients

\begin{tabular}{lcccc}
\hline Time Point & $\begin{array}{c}\text { All Assigned } \\
\text { Patients }\end{array}$ & $\begin{array}{c}\text { Coil-Assigned } \\
\mathrm{mRS}>2\end{array}$ & $\begin{array}{c}\text { Clip-Assigned } \\
\mathrm{mRS}>2\end{array}$ & p Value \\
\hline 3 yrs & 397 & $56 / 197(28.4 \%)$ & $69 / 200(34.5 \%)$ & 0.20 \\
3 yrs-CF & 411 & $56 / 203(27.6 \%)$ & $69 / 208(33.2 \%)$ & 0.24 \\
\hline
\end{tabular}

in 1997,7 stated clearly that the patient had to have an aneurysm suitable for both treatments and clinical equipoise existed for these patients. The proportion of aSAH patients enrolled in individual centers varied widely between $1 \%$ and $44 \%$, reflecting individual clinical views and belief at the time that a particular treatment was better (that is, lack of clinical equipoise). We have addressed this widespread fallacy in a recent letter published in the Journal of Neurosurgery. ${ }^{6}$

The results from BRAT are relevant in discussing this point. ${ }^{5}$ Among BRAT patients with aneurysms in the anterior circulation, $42 \%$ of the patients were deemed inappropriate for endovascular treatment and were crossed over to clipping. When those that were crossed over to clipping are compared with those that were actually treated with coil embolization, there is a dramatic difference in their outcome scores, with $23 \%$ of those treated with coils having a poor outcome (mRS score $>2$ ) compared to $42 \%$ of those who were treated with clip occlusion (p $=0.007$, Table 2). ${ }^{5}$ Yet comparison of these 2 cohorts showed no significant difference in baseline characteristics. ${ }^{5}$ As noted in our paper, "No significant differences were noted in the presentation grades or comorbidities of the patients who crossed over to surgical clipping, but the mean size of the aneurysms in the patients who crossed over was slightly smaller, and the aneurysms were more likely to be located in the anterior circulation." 5

Clearly, there were profound differences that led to the crossover of these patients. The recorded baseline characteristics obviously were not the critical factors determining whether a patient was treated with coil embolization or was crossed over; instead, decisions were made based on associated hematomas, vascular access, location and morphology of the aneurysm, or other unidentified reasons. Therefore, analyses of a subgroup of patients can only be generalized to the larger patient population with great caution.

We have always stated in all the ISAT-related publications that ISAT is a study of a selected population of aSAH patients. It is not correct to call this bias. The selected population is fully described; within that popu-

\section{TABLE 3: All deaths}

\begin{tabular}{lccccc}
\hline Time Point & $\begin{array}{c}\text { Clip-Assigned } \\
\text { Patients }\end{array}$ & $\begin{array}{c}\text { Coil-Assigned } \\
\text { Patients }\end{array}$ & Total & $\begin{array}{c}\text { Change } \\
\text { Value }\end{array}$ \\
\hline discharge & 11 & 9 & 20 & & \\
6 mos & 26 & 18 & 44 & +24 & \\
1 yr & 31 & 19 & 50 & +6 & \\
3 yrs & 35 & 25 & 60 & +10 & 0.21 \\
\hline
\end{tabular}


lation the allocation was concealed, and reporting was by intention to treat. Once patients were enrolled there was no bias in the trial. The population of patients in ISAT was balanced for known prognostic factors by the minimization process, with the allocation group revealed after the patient's baseline data had been collected. In practice, $97 \%$ of patients had anterior circulation aneurysms, $88 \%$ were in good grade (World Federation of Neurosurgical Societies [WFNS] Grade I or II), and 90\% had aneurysms less than $10 \mathrm{~mm}$ in diameter. In the United Kingdom (UK) national audit data this represented about $80 \%$ of more than 2000 patients treated between 2001 and 2002 in the UK. 4,13

The relevance of BRAT is precisely that it has addressed the entire aSAH population, with the caveat that for the anterior circulation aneurysms similar to the cohorts discussed above, $42 \%$ were found unsuitable for endovascular treatment. "Once patients were enrolled there was no bias in the trial" is open to interpretation, although this is not the appropriate forum for that discussion. However, the single fact of the more than 14 hours in delay of treatment for the clipping arm as opposed to the coiling arm in an intent-to-treat trial-when it is well known that the greatest risk of recurrent $\mathrm{SAH}$ is within the first 24 hours, ${ }^{4}$ which resulted in a large discrepancy of pretreatment SAHs and deaths in ISAT-questions the equal commitment to the trial by both specialties.

They state that the outcomes in ISAT are similar in the 2 groups at 5 years. In ISAT this was the case if the patients were alive. It ignores the fact that there was a significant excess mortality in the clip-treated group at 5 years after treatment. The similarity of outcome with respect to dependence in the report was conditional on patient survival. This was clearly stated in the 2009 Lancet Neurology paper. ${ }^{8}$ Therefore, the bald statement they make in this respect that the late outcomes are similar is not valid as stated.

This is a very valid point and we apologize for not clarifying it adequately even though it is included in the Discussion. The statement is based on the analysis of Bakker et al. ${ }^{1}$

In the Discussion, the authors reference the paper of Gnanalingham and colleagues ${ }^{3}$ as being from a major ISAT participating institution. This is incorrect, the data used for this paper were not from an ISAT recruiting center.

We apologize for this error and have corrected it; the data on delay in treatment, however, remain important and relevant.

The authors stated in their response to the letter by Drs. Darsaut and Raymond ${ }^{2}$ that although the ISAT's ratio of screened to enrolled patients was a common criticism, they "did not suggest that it invalidated the findings with respect to the population studied or even that it was a valid criticism." This current paper appears to contradict that statement. The authors stated in their letter in response to Drs. Darsaut and Raymond letter ${ }^{2}$ that the proportion enrolled in ISAT did not invalidate the findings in ISAT or even that it was a valid criticism.

At no time did we imply or believe that ISAT was not an appropriate, well-done study whose conclusions were valid for the population studied. We do believe that these results are only applicable to the cohort studied and should not be generalized to the entire aneurysm population.

\section{Comments Regarding the Discussion}

The Discussion cites O'Kelly and colleagues'll study of cases in the Ontario Stroke database involving patients treated between 1995 and 2004 as evidence that there were worse outcomes in the coil-treated patients. This presupposes that the characteristics of the population in the clip-treated and coil-treated groups have similar baseline clinical characteristics, which of course they could not have because they were not randomized. Whilst the authors attempted to stratify for initial condition in that paper and adjust statistically for the inevitable differences between the groups, there were insufficient clinical data available from an administrative database such as this to stratify for major prognostic features-notably, even clinical grade on admission. For example a significantly higher proportion of patients treated with coil embolization were ventilated on admission, and O'Kelly et al. recognized these shortcomings. Thus, attempting to draw scientifically valid conclusions from such data may be misleading.

Molyneux and colleagues are correct, and these shortcomings are well recognized. Nonetheless, the results still raise the question of overall outcomes when these treatments are applied to an SAH patient population at large.

We appreciate that the BRAT investigators have made a significant effort to address what was widely perceived to be a weakness of ISAT. BRAT was originally designed as a pilot study to test the feasibility of a larger trial, and in the report of the 1-year results, the authors stated that it was not expected to be powered sufficiently to demonstrate differences in outcome between the groups. ${ }^{5}$ However, in that report, despite the extent of the missing outcomes, the primary outcome, based on intention to treat, showed a larger absolute benefit of coiling-10\% compared with the 7.4\% that was shown in ISAT, and this difference did reach statistical significance at a $5 \%$ level $(p=0.02)$.

The current paper attempts to contradict their original findings, using subgroup analyses that have not been prespecified in a published protocol, whilst having a substantial number of missing outcomes, and attempts to draw conclusions on an inadequate sample size. It does not conform to the standard reporting criteria, set out in CONSORT criteria, whilst containing a number of factually inaccurate statements concerning ISAT. The present paper is scientifically flawed.

Our analysis of the BRAT data at 3 years does not in any way invalidate the 1-year findings; indeed they are as significant as in the first paper (Table 1). Our rationale for the 3-year analyses being restricted to those patients actually treated, as previously stated, was based on the fact that the number of crossovers could only be identified if the patients were actually available to receive treatment. We also presented data to show that this exclusion had no bearing on the results. 


\section{Editorial}

Our analysis of subgroups based on lesion location is exploratory, and this point has been clarified. We felt that separating the anterior circulation from the posterior circulation, despite limitations, provided data that were more comparable to ISAT. The fact that the anterior circulation aneurysms were well balanced in both arms and that there was no difference in outcome at any time point raises the valid question as to whether clipping should be the preferred treatment, considering the risks associated with retreatment, completeness of obliteration, and subsequent SAH risk associated with coiling.

In summary, while recognizing that the sample size leaves the study underpowered, we stand by our conclusion that there is no significant difference in an intent-totreat analysis between the 2 treatment arms at 3 years. In an ad hoc analysis of anterior circulation aneurysms performed in order to provide a cohort comparable to that of ISAT, we found no difference in outcome at any time point. A prospective trial incorporating the advances for both endovascular and microsurgical treatment remains appropriate.

\section{References}

1. Bakker NA, Metzemaekers JD, Groen RJ, Mooij JJ, Van Dijk
JM: International subarachnoid aneurysm trial 2009: endovascular coiling of ruptured intracranial aneurysms has no significant advantage over neurosurgical clipping. Neurosurgery 66:961-962, 2010

2. Darsaut TE, Raymond J: Barrow Ruptured Aneurysm Trial. J Neurosurg 117:378-379, 2012 (Letter)

3. Gnanalingham KK, Apostolopoulos V, Barazi S, O'Neill K: The impact of the international subarachnoid aneurysm trial (ISAT) on the management of aneurysmal subarachnoid haemorrhage in a neurosurgical unit in the UK. Clin Neurol Neurosurg 108:117-123, 2006

4. Kassell NF, Torner JC: Aneurysmal rebleeding: a preliminary report from the Cooperative Aneurysm Study. Neurosurgery 13:479-481, 1983

5. McDougall CG, Spetzler RF, Zabramski JM, Partovi S, Hills NK, Nakaji P, et al: The Barrow Ruptured Aneurysm Trial. Clinical article. J Neurosurg 116:135-144, 2012

6. Molyneux AJ, Kerr RS, Birks J, Ramzi N, Yarnold J, Sneade M, et al: Risk of recurrent subarachnoid haemorrhage, death, or dependence and standardised mortality ratios after clipping or coiling of an intracranial aneurysm in the International Subarachnoid Aneurysm Trial (ISAT): long-term follow-up. Lancet Neurol 8:427-433, 2009

7. Nichols DA, Brown RD Jr, Meyer FB: Coils or clips in subarachnoid haemorrhage? Lancet 360:1262-1263, 2002

Please include this information when citing this paper: published online April 26, 2013; DOI: 10.3171/2012.11.JNS121406. 